\title{
OPTICAL ANISOTROPY OF ZnSe/BeTe SUPERLATTICES PROBED BY EXCITONIC SPECTROSCOPY
}

\author{
A.V. Platonov, V.P. Kochereshko, E.L. Ivchenko \\ A.F. Ioffe Physico-Technical Institute RAS, 194021, St. Petersburg, Russia \\ D.R. Yakovlev, W. Ossau, F. Fischer, A. Waag and G. Landwehr \\ Physikalisches Institut der Universität Würzburg, 97074, Würzburg, Germany
}

Photoluminescence spectra of type-II $\mathrm{ZnSe} / \mathrm{BeTe}$ superlattices were studied. A linear polarised photoluminescence has been found in the spectral range of spatially indirect exciton transitions. This observation is interpreted in a model of optical anisotropy of heterostructures with no-common atom at interfaces.

PACS numbers: 78.55.Et, 78.66.-w, 78.66.Hf

A number of publications devoted to studies of optical anisotropy of heterostructures with no-common atom at interfaces (InAs/GaP, etc.) has been published recently [1-3]. This effect is attributed to a low local symmetry of a single interface between two different zinc-blende type structures. In heterostructures $\mathrm{CA} / \mathrm{C}^{\prime} \mathrm{A}^{\prime}(001)$ with no-common cations $\left(C \neq C^{\prime}\right)$ and anions $\left(A \neq \mathrm{A}^{\prime}\right)$, an interface consists of the two atomic planes with $A-C^{\prime}$ or $A^{\prime}-C$ bonds. In this case there exist four different types of quantum-well structures (QWs) and superlattices (SLs). The structures with similar interfaces have the $D_{2 d}$ point symmetry and are optically isotropic in the plane of interfaces. If the bonds at the left and right interfaces are different, an ideal structure with no-common atom is characterized by the in-plane anisotropy with the principal axes [110] and [110]. The microscopical reason for this in-plane anisotropy is a mixing of heavy hole and light hole states at the interfaces $[3,4]$. In this paper we present an experimental study of the in-plane optical anisotropy in novel $\mathrm{ZnSe} / \mathrm{BeTe}$ type-II quantum-well structures.

Samples were grown by MBE on (100)-oriented GaAs substrates with controlling the type of interfaces. Three types of $\mathrm{ZnSe} / \mathrm{BeTe}(100 / 50 \AA) \times 20$ samples were grown: with BeSe...BeSe, ZnTe...ZnTe and BeSe...ZnTe interfaces.

Basic optical properties of excitonic transitions in the $\mathrm{ZnSe} / \mathrm{BeTe}$ quantum well structures were reported recently in Refs. [5, 6]. The observed two photoluminescence lines with energy positions at $\approx 2.8 \mathrm{eV}$ and $\approx 2.0 \mathrm{eV}$ were related to a 
spatially direct exciton in the ZnSe layer and to a spatially indirect exciton with an electron confined in $\mathrm{ZnSe}$ and a hole confined in BeTe, respectively.

Photoluminescence measurements were performed for the temperature range 6-70 K. Photoluminescence was excited by $4416 \AA$ line of a He:Cd laser that is close to the energy of the direct exciton resonance in ZnSe layer [4]. Linearly polarized excitation along [110], [110] and [100] and circularly polarized excitation were used.

Linearly polarized photoluminescence of the indirect exciton in type-II $\mathrm{ZnSe} / \mathrm{BeTe}$ multiple quantum well structures was investigated. We have found that independently of the polarization of the excited light the resulting photoluminescence was polarized along [11̄0] direction (see Fig. 1). The degree of this

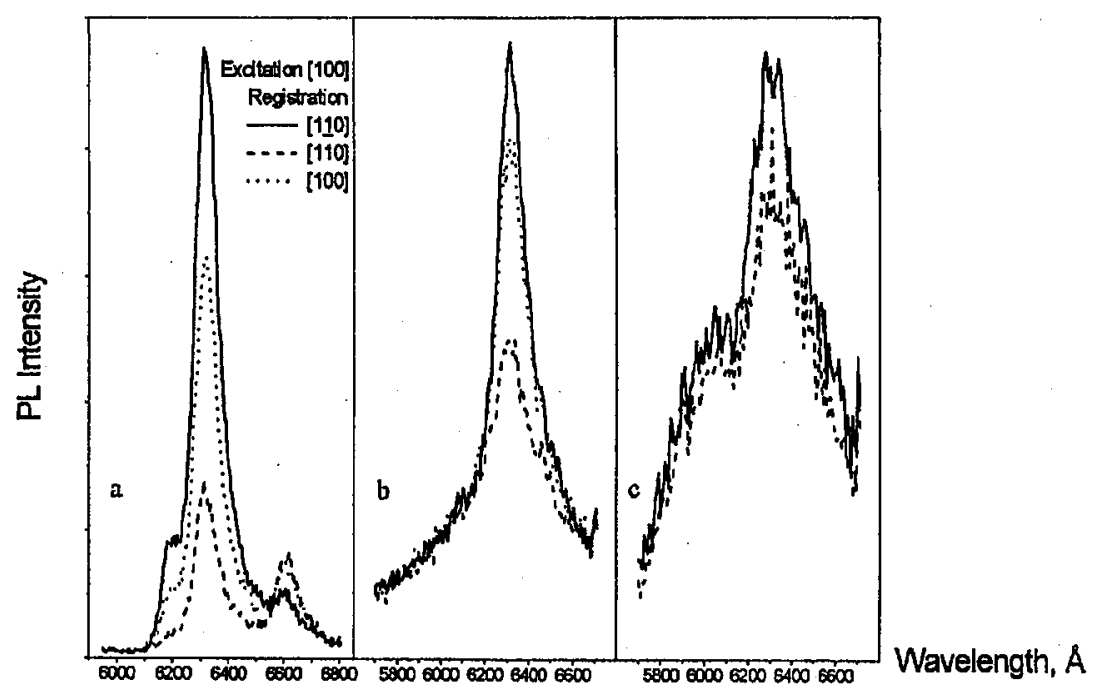

Fig. 1. Polarization resolved photoluminescence spectra intensity (arbitrary units) at the temperature $6 \mathrm{~K}$ of the $\mathrm{ZnSe} / \mathrm{BeTe} \mathrm{SLs}(100 \AA / 50 \AA)$ with the different types of the interfaces: (a) BeSe...ZnTe, (b) ZnTe...ZnTe, (c) BeSe...BeSe. Excitation is linearly polarized along [100] direction.

polarization was determined by the type of interfaces. The degree of polarization was found to be $\approx 50 \%$ for the structures with nominally equivalent interfaces $\mathrm{ZnTe} . . \mathrm{ZnTe}$ and $\approx 15 \%$ or even less for BeSe...BeSe interfaces. For the structures with nonequivalent interfaces (BeSe...ZnTe) the degree of polarization amounts to $70 \%$. At the same time we did not observe any remarkable circular polarization degree for all samples.

In the theoretical paper $[3,4]$ a generalized effective mass approximation has been used to calculate envelope functions making allowance for mixing of heavy hole and light hole states at interfaces under normal incidence. It was shown that the maximum degree of linear polarization in QWs with no-common atom at 
interfaces can reach $20 \%$, which is too small to explain the polarization degree of $70 \%$ observed in the studied structures.

To explain the large value of the polarization found experimentally one has to suggest that the hole in the indirect exciton is strongly localized at the interface. Such hole localization has been already suggested from the analysis of photoluminescence (PL) under high optical excitation conditions [7]. One of the possible mechanisms of the localization could be band bending at the $\mathrm{ZnSe}-\mathrm{BeTe}$ interface which is presented in these structures as it follows from photoemission spectroscopy [8]. To confirm this idea we have studied PL spectra of ZnSe/BeTe short-period superlattices with the layer thickness $40 \AA / 20 \AA$, where the hole wave function spreads through both interfaces. We have found no PL polarization in these structures.

It follows from symmetry analysis that in ideal structures with nominally equivalent interfaces ( $\mathrm{ZnTe} . . \mathrm{ZnTe}$ and $\mathrm{BeSe} . . \mathrm{BeSe}$ ) the linear polarization has to be absent. But for the studied structures we observed a finite value of the linear polarization degree. This effect could be related to a different electronic structure of the direct and inverted interfaces. The non-equality of the interfaces is due to a large difference between the chemical activities of the $\mathrm{Be}$ to $\mathrm{Zn}$ and $\mathrm{Se}$ to Te. This also leads to a decrease in the quality of the (ZnTe) as well as (BeSe) interfaces on the BeTe layer. At the same time on the ZnSe layer both interfaces can be grown of high quality. A poor quality of the interface should lead to the highest nonradiative recombination and to a different contribution to integral $\mathrm{PL}$ signal from the direct and inverted interfaces. This reason should lead to the observed linear polarization of the structures with nominally equivalent interfaces. Another reason which decreases the symmetry of the structure and makes possible to observe the in-plane anisotropy could be a built-in electric field [3]. For the studied structures we can except a presence of the field due to the band bending which was discussed before.

We also measured the temperature dependence of the PL intensity. With the temperature increasing the $\mathrm{PL}$ intensity is rapidly suppressed while the polarization degree is constant.

In summary we have presented the results of the polarization-resolved $\mathrm{PL}$ studies of the type-II SLs ZnSe/BeTe. We found that PL signal from spatially indirect exciton is strongly polarized along [1T0] direction. This effect is ascribed to the general in-plane anisotropy of the heterostructures with no-common atoms at the interfaces. The high value of the polarization degree corresponds to the strong hole localization at the interfaces in the type-II heterostructures.

The work was supported in part by RFBR grant No. 98-02-18234, the program "Nanostructures" of Russian Ministry of Sciences and the Deutsche Forschungsgemeinschaft (SFB 410).

\section{References}

[1] O. Krebs, P. Voisin, Phys. Rev. Lett. 77, 1829 (1996).

[2] O. Krebs, W. Seidel, P. Voisin, in: Proc. 23rd Int. Symp. Compound Semiconductors, St. Petersburg 1996, Eds. M.S. Shur, R.A. Suris, Inst. Phys. Conf. Ser. No. 155, IOP Publ., Singapore 1997, Ch. 12, p. 859. 
[3] E.L. Ivchenko, A.A. Toropov, P. Voisin, Phys. Solid State, 1998, to be published.

[4] E.L. Ivchenko, A.Yu. Kaminskii, U. Rössler, Phys. Rev. B 54, 5852 (1996).

[5] A.V. Platonov, D.R. Yakovlev, U. Zehnder, V.P. Kochereshko, W. Ossau, F. Fischer, T. Litz, A. Waag, G. Landwehr, Acta Phys. Pol. A 92, 1063 (1997).

[6] A.V. Platonov, D.R. Yakovlev, U. Zehnder, V.P. Kochereshko, W. Ossau, F. Fischer, T. Litz, A. Waag, G. Landwehr, J. Cryst. Growth 184/185, 801 (1998).

[7] S.V. Zaitsev, V.D. Kulakovskii, A.A. Maksimov, D.A. Pronin, I.I. Tartakovskii, N.A. Gippius, D.R. Yakovlev, W. Ossau, G. Landwehr, JETP Lett. 66, 376 (1997).

[8] M. Nagelstrasser, H. Droge, F. Fischer, T. Litz, A. Waag, G. Landwehr, H.-P. Steinruck, J. Appl. Phys. 83, 4253 (1998). 